\title{
Warm dense matter created by isochoric laser heating
}

Y. Ping, A. Correa, T. Ogitsu, E. Draeger, E. Schwegler, K. Widmann, D. F. Price, E. Lee, H. Tam, P. T. Springer, D. Hanson, I. Kowlow, D. Prendergast, G. Collins, A. Ng

November 5, 2009

International Conference on High Energy Density Physics austin, TX, United States

May 19, 2009 through May 22, 2009 
This document was prepared as an account of work sponsored by an agency of the United States government. Neither the United States government nor Lawrence Livermore National Security, LLC, nor any of their employees makes any warranty, expressed or implied, or assumes any legal liability or responsibility for the accuracy, completeness, or usefulness of any information, apparatus, product, or process disclosed, or represents that its use would not infringe privately owned rights. Reference herein to any specific commercial product, process, or service by trade name, trademark, manufacturer, or otherwise does not necessarily constitute or imply its endorsement, recommendation, or favoring by the United States government or Lawrence Livermore National Security, LLC. The views and opinions of authors expressed herein do not necessarily state or reflect those of the United States government or Lawrence Livermore National Security, LLC, and shall not be used for advertising or product endorsement purposes. 


\title{
Warm dense matter created by isochoric laser heating
}

\author{
Y. Ping ${ }^{1}$, A. A. Correa ${ }^{1}$, T. Ogitsu ${ }^{1}$, E. Draeger ${ }^{1}$, E. Schwegler ${ }^{1}$, T. Ao ${ }^{2}$, K. Widmann ${ }^{1}$, D. F. Price ${ }^{1}$, E.

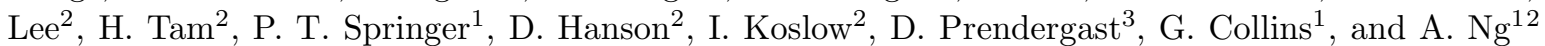 \\ ${ }^{1}$ Lawrence Livermore National Laboratory, \\ Livermore, CA, U.S.A. \\ ${ }^{2}$ Department of Physics \& Astronomy, \\ University of British Columbia, \\ Vancouver, B.C., Canada \\ ${ }^{3}$ The Molecular Foundry, \\ Lawrence Berkeley National Laboratory, \\ Berkeley, CA, U.S.A.
}

(Dated: November 5, 2009)

\begin{abstract}
Warm Dense Matter (WDM) physics has been a growing field as part of high energy density physics, driven by the fundamental urge to understand the convergence between plasma and condensed matter physics, and the practical need to understand dynamic behavior of material under extreme conditions. A platform for creating and probing WDM by isochoric heating of free-standing nano-foils has been developed recently to study the non-equilibrium processes. Results of optical measurements reveal the existence of a quasi-steady state in the time history, during which the interband component of the dielectric function shows both enhancement and red shift. First principle calculations of the dielectric function suggest that the enhanced red-shift of interband transition peak might be explained by a positive charge state of gold foil due to ejection of electrons by a high intensity laser pulse. The impact of electronic sheath formation at the surface on the optical property was examined by the Thomas-Fermi theory with local equilibrium approximation.
\end{abstract}

PACS numbers: 52.50.Jm, 52.25.Fi, 52.25.Os, 52.38.--r

\section{INTRODUCTION}

Warm dense matter lies in a regime where densities are near the solid density and temperatures are between 0.1 and $100 \mathrm{eV}$. These warm and dense states are characterized by comparable thermal and Fermi energies of electrons and strongly correlated ionic cores [1] . At the crossroad between condensed matter physics and plasma physics, warm dense matter are very difficult to model because they involve degenerate, strongly-coupled states which evolve through non-perturbative, non-adiabatic processes. On the other hand, the high-energy-density nature of warm dense matter makes experimental study also challenging. Data obtained from expanding systems would be integrated over mixed states, thus difficult to interpret. This has left warm dense matter a largely uncharted frontier. Nonetheless, warm dense matter is drawing increasing attention because of its role in understanding the convergence between condensed matter and plasma physics, as well as its relevance to other areas including shock physics [2], inertial confinement fusion [3] and astrophysics [4]. Among this broad interest, a new focus is non-equilibrium warm dense matter. This is driven by its practical importance as transient states in laboratory-produced high energy density matter and its fundamental significance in the study of relaxation processes and phase transitions.

A platform for creating and probing WDM by isochoric laser heating of nano-foils has been developed recently $[5,6]$. In this scheme, a freestanding ultrathin gold foil is irradiated by fs laser pulses. The thickness of the foil is chosen to be shorter than the ballistic distance of excited electrons so that the heating is mostly uniform in the longitudinal direction. The ultrafast heating by fs laser pulse creates highly excited electrons semiinstantaneously, leaving the lattice virtually in an unperturbed cold state. Therefore a non-equiliibrium state of warm dense matter is initiated. Due to the small electron-phonon coupling of gold, the lattice heating by the excited electrons is expected to be slow in the laser heated nano-gold foil. The optical measurements are performed before the transition to liquid and hydrodynamic expansion in order to probe the properties of warm dense gold with minimum spatial gradients. A key parameter characterizing warm dense matter, the dielectric function including both the intraband (Drude) region and interband region, has been measured for isochorically heated gold foils. The results have revealed some novel phenomena and properties of the warm dense gold, such as a quasi-steady-state behavior in the time history [7], and enhanced interband transition [8].

This paper is a review of the experimental results, as well as theoretical efforts to understand the exotic behaviors. Development of theoretical models in this regime is challenging due to non-perturbative and non-adiabatic nature of WDM. As seen in Fig. 1, WDM regime appears as a critical void in the equation of state (EOS) table of $\mathrm{Cu}$ as calculated by six models [9]. Recent advancement in computational capabilities has enabled substantial progress in first-principle simulations of a warm dense system. Ab initio calculation of the dielectric function of aluminum across the solid-liquid transition has 


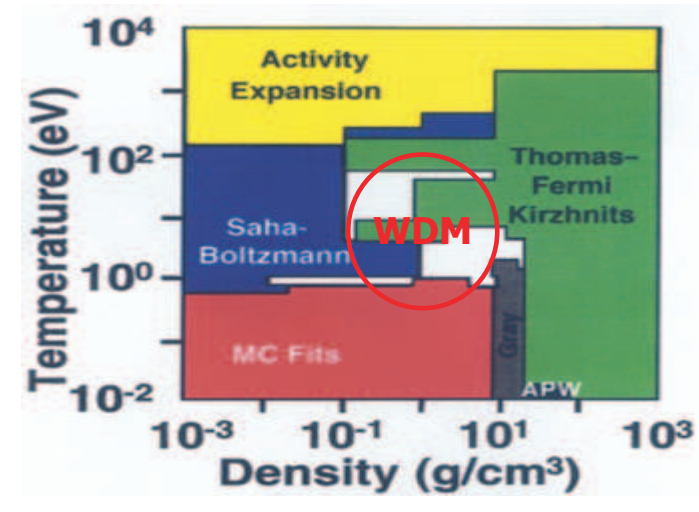

FIG. 1: Temperature-density map showing the subregions covered by six theoretical models for calculations of EOS of $\mathrm{Cu}$ (from [9]).

been obtained [10, 11]. Calculation of ac conductivity of warm dense gold in a spectral range that covers both intra-band and inter-band transitions has been reported albeit based on very limited Brillouin zone sampling [12].

The paper is organized as follows. The Fourier domain interferometry (FDI) and broadband measurements are presented in Sec. II and III, respectively. A possible nonneutral scenario is explained in Sec IV, and calculations of the electron sheath formation is described in Sec. V. Finally the summary is given in Sec. VI.

\section{EXPERIMENTAL METHOD AND FDI MEASUREMENTS}

The experiment was performed on the Europa laser at Jupiter laser Facility (JLF) at LLNL. The targets were free-standing gold nanofoils (25-33 nm thick), as shown in Fig. 2. The flatness of the foil was critical for optical measurements. The foil surface quality was checked using a Michelson interferometer with a $\mathrm{HeNe}$ laser at $632.8 \mathrm{~nm}$, indicating flatness better than $\lambda / 10$ over a $300-$ $\mu \mathrm{m}$ region as shown in Fig. 2(a) and (d). The central flatness within the pump focal size was comparable with a $\lambda / 20$ optical mirror, which enabled high-quality optical reflectivity measurements.

Figure 3 shows the experimental pump-probe schematic. A 150-fs FWHM, frequency-doubled laser pulse provided the pump for isochoric heating of the gold foils. The 400-nm pump pulse was focused onto the nanofoil at normal incidence with a spot diameter of 80 $\mu \mathrm{m}(\mathrm{FWHM})$. The incident $\left(E_{i n}\right)$, reflected $\left(E_{r}\right)$ and transmitted $\left(E_{t}\right)$ pump light were monitored by both calibrated photodiodes with integrating spheres and CCD cameras to yield measurements of laser deposition across the focal spot with a spatial resolution of $5 \mu \mathrm{m}$. While the skin-depth of 400-nm light is only $\sim 7 \mathrm{~nm}$ in gold, the electron ballistic range is $\sim 110 \mathrm{~nm}$ [13] which far exceeds the thickness of the target. Combined with the 150 -fs pulse width, this results in uniform and
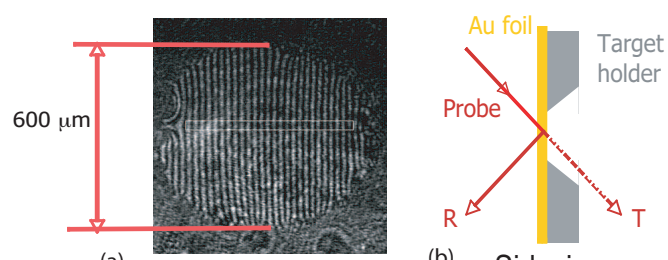

(a)

(b) Sideview
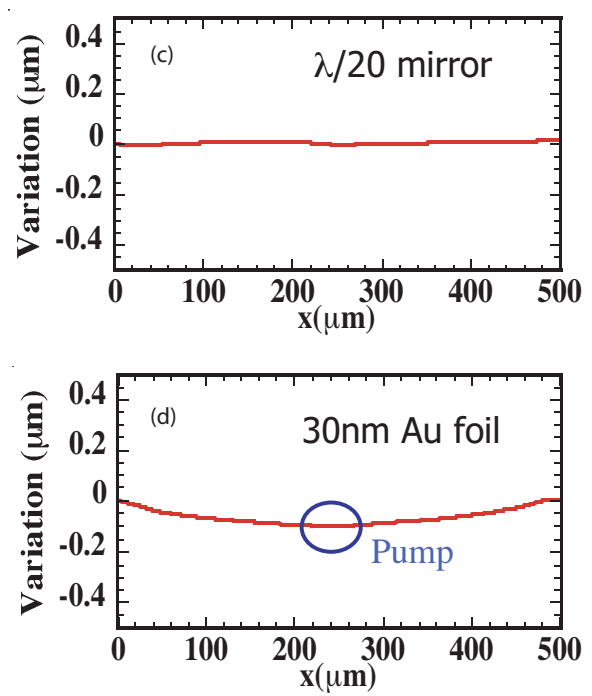

FIG. 2: (a) Fringe pattern across the foil surface. (b) Side view of the free-standing foil. (c) and (d) are surface variation of a $\lambda / 20$ mirror and the gold foil over a $500-\mu \mathrm{m}$ region.

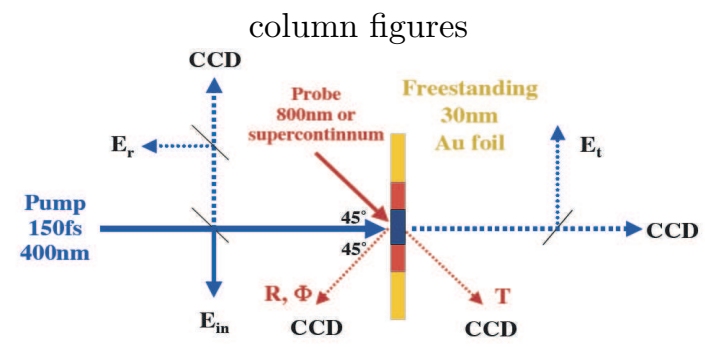

FIG. 3: Experimental schematic.

isochoric heating of the nanofoil at solid density and allows the determination of excitation energy density $E_{D}$ directly from the laser deposition measurement. The energy density cited in this paper is the average over the central $15-\mu \mathrm{m}$ region where the deposition of the pump pulse was uniform. The probe was incident onto the target at $45^{\circ}$. The reflected and transmitted probe were directed into spectrometers with a CCD camera to allow for either FDI or broadband measurements.

Fourier domain interferometry (FDI) was employed to measure the phase shift of an $800 \mathrm{~nm}$ probe reflected from the laser-heated gold foil. The setup for FDI is illustrated in Fig. 4 (top panel). The probe was split into two identical pulses traveling with a delay between them. The first one reflected off the target before the pump pulse, thus providing a reference phase. The second one inter- 

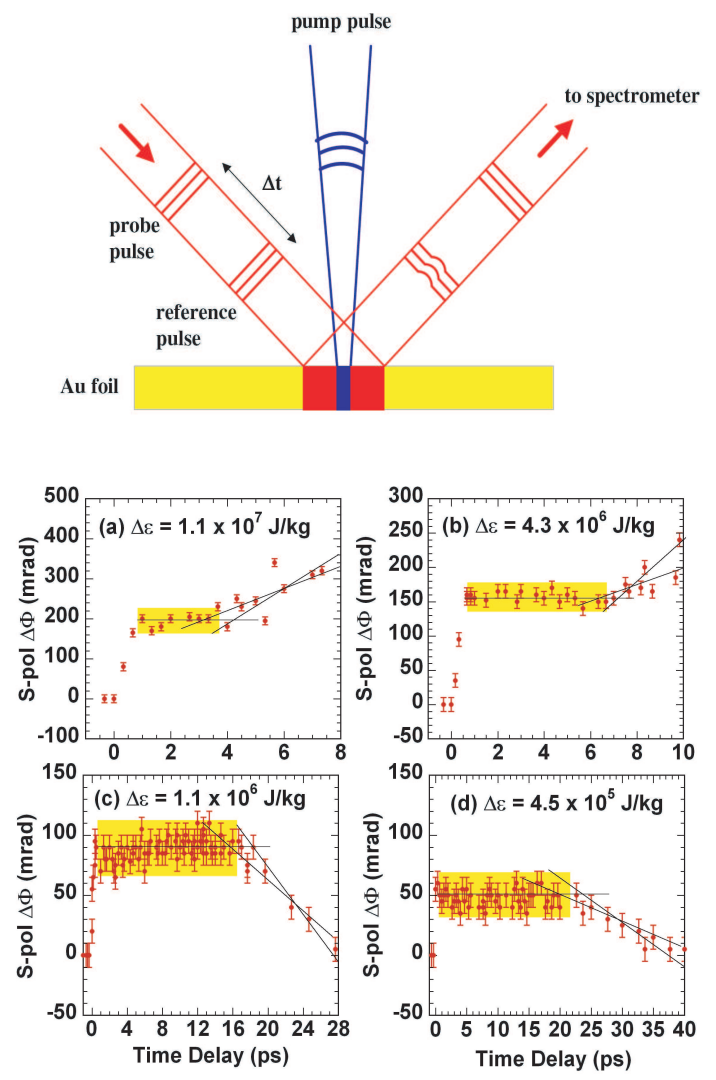

FIG. 4: Top: Illustration of Fourier domain interferometry. (a)-(d) Phase shift as a function of time for four different energy densities. The quasi steady state behavior (yellow shade) is observed at all the energy densities. The error bars are uncertainties due to the noise level of the measurement. The solid lines are guides for eyes and are to illustrate how the steady state duration $(\Delta \tau)$ in Fig. 5 was determined.

acted with the target after the heating pulse and hence carried a phase shift relative to the reference. In the spectrometer the delay between the two pulses was partially compensated by the dispersion due to the grating. Therefore the two pulses could overlap in time and interfere to create fringes in the frequency domain. The minimum phase shift that can be measured by this setup is $\sim 10 \mathrm{mrad}$, corresponding to a spatial sensitivity of $13 \AA$.

The measured phase shifts of a s-polarized probe by FDI as a function of time for four energy densities are displayed in Fig. 4 (a)-(d). At all the energy densities, the quasi- steady state (QSS) behavior is clearly observed. The duration of QSS varies as a function of pulse laser energy density: the higher the energy density, the shorter the QSS. Quantitatively, the QSS decreases from 20ps to $1.7 \mathrm{ps}$ as the energy density increases from $5 \times 10^{5}$ to $1.5 \times 10^{7} \mathrm{~J} / \mathrm{kg}$, as plotted in Fig. 5 . Such a time scale can be compared with ultrafast electron diffraction (UED) measurements by Dwayne Miller's group on laser heated gold foils [15], where the lattice dynamics for a range of energy densities similar to our study were reported. It

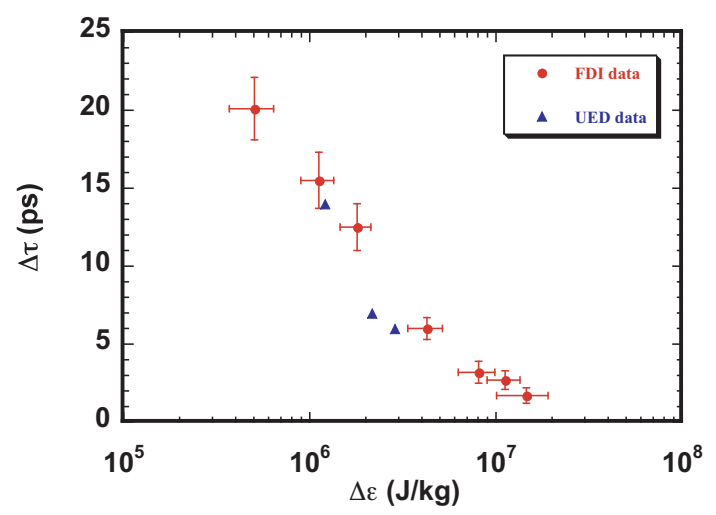

FIG. 5: The duration of the quasi-steady state vs. energy density (red points). Also shown is the time for Debye-Waller factor of the (220) diffraction peak to drop to $10 \%$ of its room temperature value by UED measurements in laser-heated gold foils (data from [15]).

is found that the durations for the Debye-Waller factor of the (220) diffraction peak to decay to the $10 \%$ of its room temperature value agree quantitatively with the QSS durations (see Fig 5). We point out that there are some difference in the experimental conditions, for example, the thickness of foil (FDI: $\mathrm{d}=30 \mathrm{~nm}$; UED: $\mathrm{d}=20 \mathrm{~nm}$ ) and the wavelength of the pump laser (FDI: $\lambda=400 \mathrm{~nm}$; UED: $\lambda=387 \mathrm{~nm}$ ), which might affect on the lifetime of those states of gold created by laser pulses. Nevertheless, the both measurements indicate that those pumped gold foils retain the $f c c$ crystal order in its atomic configurations to some extent during those durations. The QSS behavior in optical properties, in contrast to the continuously varying lattice dynamics as shown in UED measurements, remains to be understood. However, our work demonstrates that the temporal signatures render FDI a plausible means of probing time scales of structural phases.

The quasi-steady state behavior supports the validity of our assumption used in the analysis of our data; the gold foil does not expand during the QSS. The phase shift is determined by both dielectric function of the target and motion of the critical density layer when hydrodynamic expansion occurs. Therefore, if the hydrodynamic expansion is taking place during the QSS, it would require the spatially varying dielectric property of an expanding foil to evolve in such a manner to mitigate precisely effects due to density gradient or hydrodynamic flow for the entire duration of the quasi-steady state. This is highly unlikely. Therefore, it is reasonable to assume that the gold foil maintains its slab-like geometry, which enable us to calculate the evolution of the dielectric function of the warm dense gold from reflectivity and transmissivity measurements of a probe. In the FDI experiment, the photon energy of the probe is $1.55 \mathrm{eV}(800 \mathrm{~nm})$, which is below the interband transition in Au. Therefore, the FDI data present the intraband part of the dielectric function. Based on the Drude formula [14], the DC conductivity, 
column figures

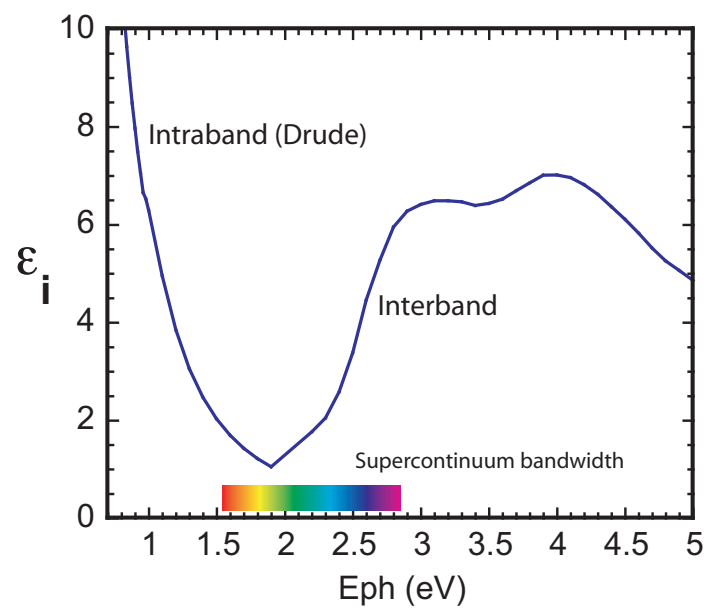

FIG. 6: Dielectric function of $\mathrm{Au}$ from $0.7 \mathrm{eV}$ to $5.0 \mathrm{eV}$ at ambient conditions, showing both the intraband and interband components (data from [17]). The bandwidth of the supercontinuum probe is marked.

free electron density and collision frequency can be extracted, which has been discussed in detail in Ref.[6-8].

\section{BROADBAND MEASUREMENTS}

To study not only the intraband but also the interband components of the dielectric function, broadband measurements were performed using a supercontinuum probe. For $\mathrm{Au}$ there is a d-s/p transition above $\sim 2.3 \mathrm{eV}$, as seen in Fig. 6. The supercontinuum probe was generated by focusing a 180 -fs FWHM, 800-nm pulse onto a $\mathrm{CaF}_{2}$ crystal. Its spectrum extends from 1.50 to 2.85 $\mathrm{eV}$, covering both intraband and interband regions, as indicated in Fig. 6. Details of the supercontinuum setup and characterization can be found in [16].

The temporal evolution of the dielectric function of the warm dense gold, $\varepsilon(\omega)$, at an excitation energy density of $(2.9 \pm 0.3) \times 10^{6} \mathrm{~J} / \mathrm{kg}$ is shown in Fig. 7. Time zero corresponds to the on-set of changes in probe reflection and transmission from their room temperature values. This is found to be the same for all frequencies in the supercontinuum spectrum after chirp correction. Also included in the figure are tabulated data of gold at room temperature [17]. The time span is chosen to cover an initial transient followed by a quasi-steady state as discovered in the earlier studies $[6,7]$. At a photon energy of $1.55 \mathrm{eV}$, the dielectric function shows an initial transient consisting of a decrease (increase) in the real (imaginary) part to a minimum (maximum) value at $\sim$ $0.8 \mathrm{ps}$, then followed by an increase (decrease) to a quasisteady-state value at $\sim 1.2 \mathrm{ps}$. This is consistent with the phase shift measurements [7]. A single $\varepsilon(\omega)$ plot is presented for 1.2-4.0 ps since no significant change in $\varepsilon(\omega)$ is observed in the interval. The existence of a quasi-steady state for the observed spectral range is consistent with

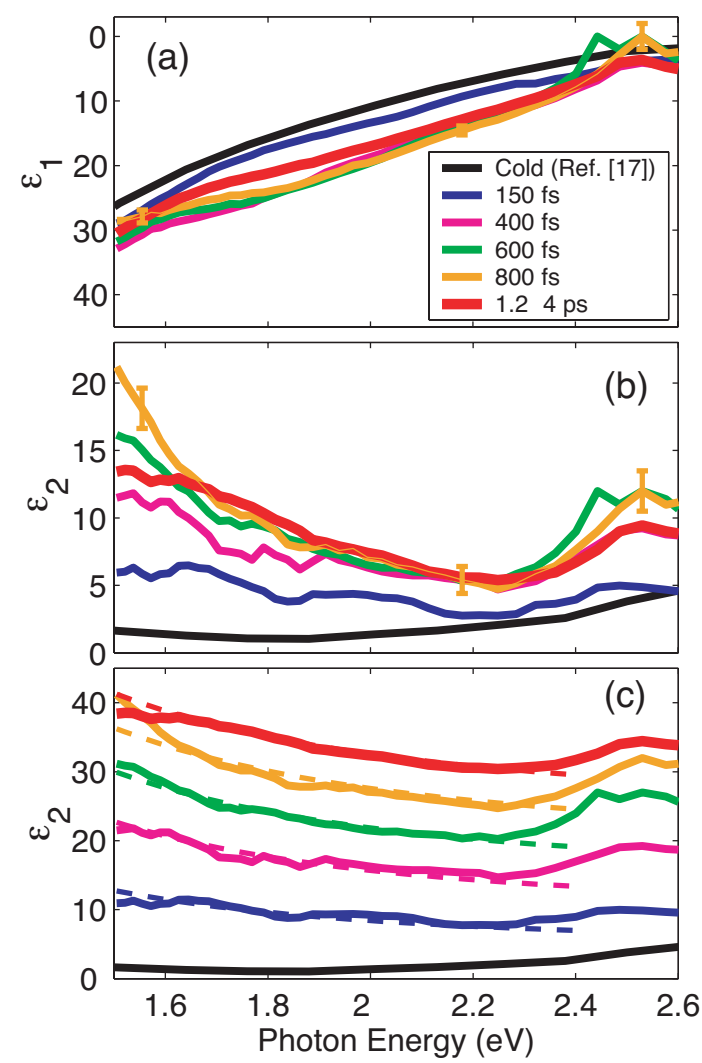

FIG. 7: (a) $\varepsilon_{1}(\omega)$ and (b) $\varepsilon_{2}(\omega)$ at different times for an energy density of $2.9 \times 10^{6} \mathrm{~J} / \mathrm{kg}$, and (c) $\varepsilon_{2}(\omega)$ displayed with an off-set of +5 along the $y$-axis between time steps. Sample error bars are included in (a) and (b). The dashed curves are best-fit Drude functions [8].

the phase shift data, confirming the validation of ISP geometry as discussed in Ref. [7]. From photon energies $1.55 \mathrm{eV}$ to $2.60 \mathrm{eV}, \varepsilon_{1}(\omega)$ appears relatively featureless. However, intra-band and inter-band $(d-p)$ components are clearly discernable in $\varepsilon_{2}(\omega)$ below and above $\sim 2.3$ $\mathrm{eV}$. They both show substantial enhancements over their room- temperature values. Furthermore, by displaying $\varepsilon_{2}(\omega)$ at different time steps with an offset (Fig. 7(c)), it can readily be seen that the intra-band component shows good agreement with best-fitted Drude functions that assume frequency-independent electron collision time and density.

To examine the dependence of $\varepsilon(\omega)$ on the excitation energy density $E_{D}$, we use measurements made on the quasi-steady state. The results are presented in Fig. 8. These are not corrected for frequency chirp and the probe delay varies from $1.4 \mathrm{ps}$ at $1.55 \mathrm{eV}$ to $2.0 \mathrm{ps}$ at $2.6 \mathrm{eV}$. For $E_{D}$ of $2.2 \times 10^{6}$ and $4.7 \times 10^{6} \mathrm{~J} / \mathrm{kg}$, the $1.4-2.0$ ps probe delay falls completely within the quasi-steady state duration [7] allowing the dielectric function to be determined over the entire spectral range of 1.5-2.7 eV. However, at $1.7 \times 10^{7} \mathrm{~J} / \mathrm{kg}$ no acceptable solution can be found for $\varepsilon(\omega)$ from the $R, T$ data above $2.38 \mathrm{eV}$. This is due to the breakdown of the uniform slab assumption as target 

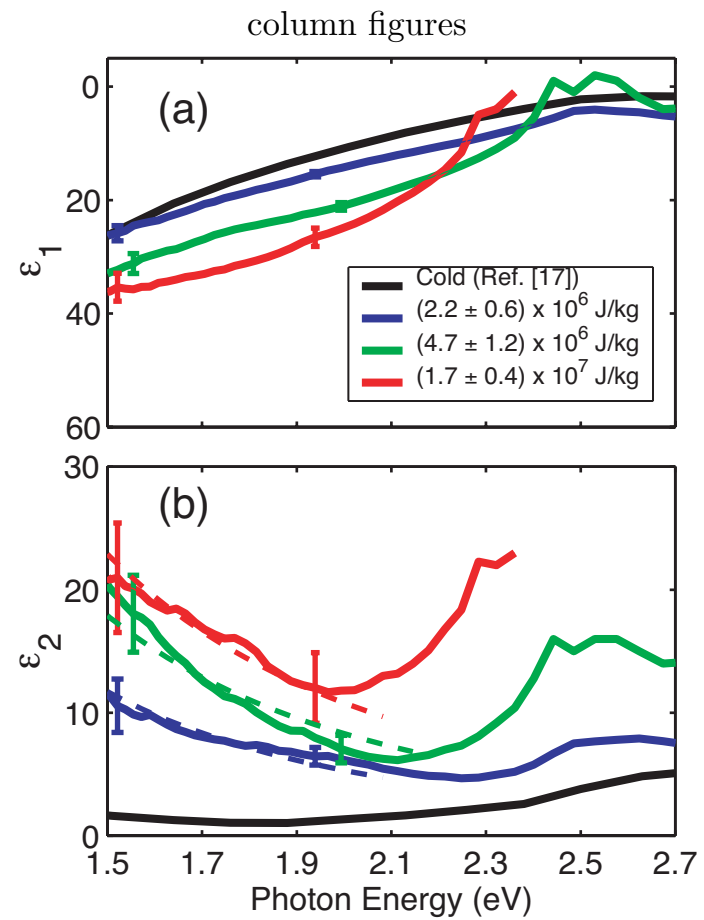

FIG. 8: (a) $\varepsilon_{1}(\omega)$ and (b) $\varepsilon_{2}(\omega)$ for different excitation energy densities. Sample error bars are included. The dashed curves are best-fit Drude functions [8].

disassembly gives rise to gradients in the expanding foil. The cut-off point at $2.38 \mathrm{eV}$ corresponds to a probe delay of 1.9 ps that is consistent with the disassembly time observed in the earlier experiment [7]. Accordingly, dielectric function measurement using a frequency chirped source offers a new means of probing hydrodynamic disassembly of a heated solid.

With increasing excitation energy density, the quasisteady-state $\varepsilon_{1}(\omega)$ begins to reveal the effect of interband $(d-p)$ transitions above $2.1 \mathrm{eV}$ while $\varepsilon_{2}(\omega)$ exhibits increasing enhancements in intra-band and inter-band transitions. The intra-band component continues to be Drude-like as indicated by the best-fit Drude functions in the figure. It should be noted that the quasi-steady state collision time and electron density are in good agreement with previous single-wavelength measurements at $800 \mathrm{~nm}$ $(1.55 \mathrm{eV})[6]$. The inter-band component also shows a red shift that increases with excitation energy density.

A basic property contained in the spectral dielectric function is electronic joint density of state. Thus, the data in Fig. 7 carry information on the evolution of the density of the $d$ and $s / p$ states driven by the processes of photo-excitation of $d$-electrons, electron-hole recombination and electron-electron thermalization at high energy densities. Similarly, the data in Fig. 8 carry information on the dependence of density of state on excitation energy density. For the range of conditions of interest, Drudelike behavior of the intra-band component of $\varepsilon_{2}(\omega)$ is confirmed. This provides a crucial validation for the use of ac conductivity at $800 \mathrm{~nm}$ to derive dc conductivity, collision time and carrier density of warm dense gold [6], thus significantly enhancing the utility of ac conductivity measurement.

The observed inter-band transition peak in the QSS presents the first evidence of persistence of the long range ordering in warm dense gold. It is generally known that a peak in $\varepsilon_{2}(\omega)$ originates from a pair of parallel bands separated by near constant energy. If the system becomes completely disordered, it is expected that the band structure will be disturbed, and the peaks in the dielectric function will be smeared out. Therefore, one could speculate that the observed enhanced inter-band transition peak reflects the presence of long-range ordering of ions, and that the quasi-steady state is the characteristic of a (heated) solid which would be consistent with a recent report based on an empirical two- temperature model [12] and UED measurements [15]. Equally important are the observed increases in red shift and enhancement of interband transition peak with excitation energy density. Calculated optical absorption spectrum of equilibrium solid and liquid phases of aluminum $[10,11]$ show red shifts in inter-band transition peak with significant smearing as the temperature increases, which is consistent with observation [18]. In this case, the mechanism of red-shift could be understood as the scattering of those parallel bands due to the thermal disorder, which will lead to effectively lower interband transition onset (red shift) at the expense of broadening of the peak. What was observed in the $\varepsilon_{2}(\omega)$ of pumped gold foil is the clear red shift and enhancement of the interband transition peak, which cannot be explained by the scattering of band structure due to thermal disorder. it should be noted that, those calculations of optical property of heated aluminum were performed at thermal equilibrium conditions, i.e., $T_{\text {ele }}=T_{i o n}$, while $T_{\text {ele }} \gg T_{i o n}$ is expected in our experiments. In order to understand the origin of the red shift and the enhancement of interband transition peak at highly non-equilibrium condition, we performed first-principle simulations as discussed below.

\section{IV. $A B$ INITIO CALCULATIONS OF $\varepsilon_{2}(\omega)$}

In this section, we describe our computational procedure, together with the actual physical problem, for calculating the time evolution of the optical absorption of gold induced by a femto-second laser pulse. In this procedure, we use the same assumptions taken in the twotemperature model. We first present our results and their interpretation and then discuss further implications.

Our problem of interest is as follows. At $t=0$, the photon pulse $(\hbar \omega=3.1 \mathrm{eV}$ with a pulse width of $150 \mathrm{fs})$ hits the gold foil $(\mathrm{d}=30 \mathrm{~nm})$, and excites the electrons in the occupied bands. Since the top of the $5 d$ band of gold lies $\sim 2 \mathrm{eV}$ below the Fermi level [19], a $3.1 \mathrm{eV}$ photon is able to excite an electron in $5 d$ band directly into an unoccupied conduction band. Note that at the pump laser fluences that were used in the experiments 
[6-8], the number of absorbed $(3.1 \mathrm{eV})$ photons reaches about one photon per gold atom. In other words, the initial electronic excitation is a massive perturbation of the electronic system. The excited electrons relax via electron-electron scattering and electron-phonon scattering, and, due to this latter inelastic channel, the lattice temperature increases[20].

Here we introduce the first assumption: The relaxation time scale within the electronic degrees of freedom is significantly shorter than the electron-phonon relaxation time scale, so that the electronic degrees of freedom are always close to thermal equilibrium, with the electronic temperature decreasing relatively slowly due to energy dissipation into the lattice via inelastic electron-phonon scattering. The time evolution of the ionic degrees of freedom will be manifest as a gradual increase in thermal disorder, whose rate is determined by the electronphonon coupling constant.

The above approximations enable us to calculate the time evolution of $\varepsilon_{2}(\omega)$ in the following way. First, the time evolution of the electron temperature and lattice temperature can be calculated using the two-temperature model[20], where only the following information is required: the specific heat of electrons, the specific heat of lattice, the electron-phonon coupling constant, and the absorbed energy density from the pump laser pulse. Then, atomic configurations corresponding to the ionic temperature for the given time are generated by ab-initio molecular dynamics simulations. Finally, the $\varepsilon_{2}(\omega)$ is calculated from the electronic structure associated with those atomic configurations.

For these simulations, we do not take the presence of surface into account. In the other words, all of the simulations presented in this section represent bulk gold. A possibly more realistic system, a slab of gold in vacuum, can be simulated in principle, however, such calculations of $\varepsilon_{2}(\omega)$ of hot gold foil with $\mathrm{d}=30 \mathrm{~nm}$ in vacuum at elevated electron temperatures are currently intractable. Instead, we examined the effect of gold thickness as well as of the surface using the Thomas-Fermi model with a local equilibrium approximation, which is presented in the next section. In short, within those approximations, the thickness of gold foil $(\mathrm{d}=30 \mathrm{~nm})$ and the presence of surface does not qualitatively affect the results presented in this section using bulk models for gold.

The specific heat of electrons was calculated from the Electronic Density of States (EDOS) using firstprinciples Density Functional Theory (DFT)[21] within the Local Density Approximation (LDA)[22], for an fcc atomic lattice at $\mathrm{T}=0 \mathrm{~K}$. We used a Troullier-Martins norm-conserving non-local pseudopotential[23] of separable form[24] for the ionic potential of gold. The $6 s, 6 p$, and $5 d$ states were included explicitly as valence states, with the $6 S$ channel chosen for the local potential[25]. Planewave expansions with $E_{c u t}=80$ Ry were used to described the electronic wavefunctions.

The pseudopotential was tested with respect to reproduction of the electronic band structure (see Fig.

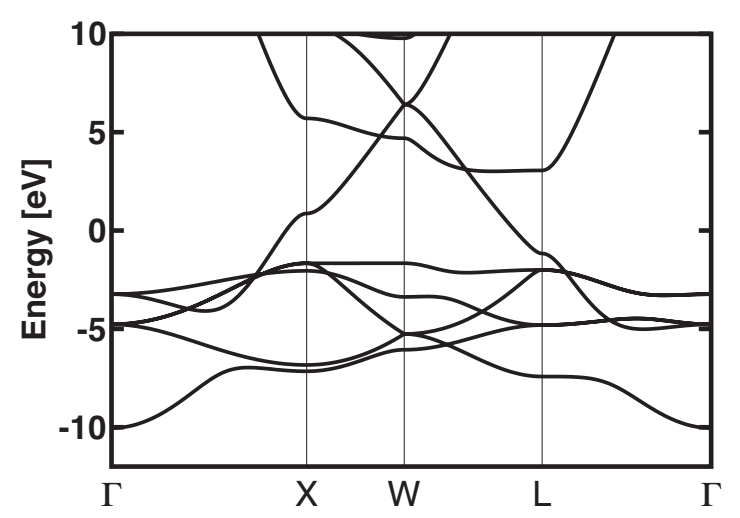

FIG. 9: The calculated electronic band structure of $f c c$ gold. The Fermi level is set to be $0 \mathrm{eV}$.

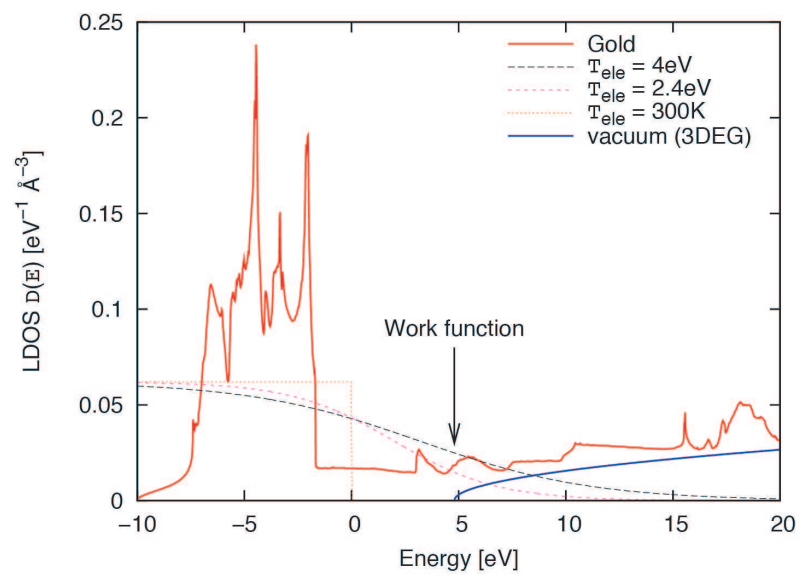

FIG. 10: EDOS of $f c c$ gold and the Fermi distribution functions at $T_{\text {ele }}=300 \mathrm{~K}, T_{\text {ele }}$ corresponding to $2.4 \mathrm{eV}$ and $4.0 \mathrm{eV}$. The Fermi level at $T_{e l e}=0 \mathrm{~K}$ is set to be $0 \mathrm{eV}$, and the workfunction of gold is $+4.8 \mathrm{eV}$ indicated by the vertical arrow. Free electron gas (3DEG) density of states of vacuum is plotted above the work function.

$9)$ and the equilibrium lattice constant $\left(a_{D F T}=7.68 \mathrm{au}\right.$, $\left.a_{\text {exp. }}=7.71 \mathrm{au}\right)$. Overall good agreement with the available data was confirmed. To calculate the EDOS, we used the PWSCF code[26] where the tetrahedron integration scheme was employed on a $32 \times 32 \times 32$ grid of $\mathrm{k}$ points in the First Brillouin Zone (FBZ). The calculated EDOS is shown in the Fig. 10.

From the transmission and reflection measurements, the amount of energy absorbed by the gold foil was estimated. For the absorbed energy, we used a value normalized by the mass of gold, $E_{D}(\mathrm{~J} / \mathrm{Kg})$. We assumed that the absorbed energy in the target area is instantaneously spread out homogeneously through the ballistic diffusion of conduction electrons [27]. Using the electronic specific heat calculated from EDOS, the initial electron temperature at $E_{D}=2.9 \mathrm{MJ} / \mathrm{kg}$ has been estimated to be 
$T_{\text {ele }}=2.4 \mathrm{eV}$.

The atomic configurations corresponding to the initial state, $T_{i o n}=300 \mathrm{~K}$ were generated using BornOppenheimer ab-initio molecular dynamics[28] with the calculation conditions mentioned above, except that our 32 atom supercell calculations employed Chadi and Cohen's two special $k$-points for the $f c c$ lattice in order to sample the FBZ[29]. Taking advantage of the large atomic mass of gold $(\sim 196.967)$, a relatively large MD time step, $\delta t=256$ au $(\sim 6 \mathrm{fs})$, was used. After several picoseconds of $\mathrm{MD}$ simulation at $T=300 \mathrm{~K}$, a some equilibrated atomic configurations were sampled and the imaginary part of dielectric function was calculated using the Kubo-Greenwood formula[30]:

$$
\varepsilon_{2}\left(\omega, T_{\text {ele }}\right)=\frac{8 \pi^{2} e^{2}}{\Omega \omega^{2}} \sum_{c, v, \mathbf{k}}|\rangle v, \mathbf{k}|\hat{p}| c, \mathbf{k}\left\langle\left.\right|^{2}\right.
$$

$$
\times \delta\left(E_{c, \mathbf{k}}-E_{v, \mathbf{k}}-\hbar \omega\right)\left[f\left(E_{v, \mathbf{k}}, T_{e l e}\right)-f\left(E_{c, \mathbf{k}}, T_{e l e}\right)\right] .
$$

As demonstrated in previous work [8, 11], to obtain a well converged profile for the dielectric function requires a very fine $k$-point sampling grid, and the calculation cost and the memory space for the eigenfunctions could become unaffordable even for simple elemental solids such as aluminum and gold. Here, we employed an improved implementation[31] of the interpolation scheme originally developed by Shirley [32].

This method was already used to calculate the optical conductivity of aluminum at wide range of temperature and pressure[11], and demonstrated good agreement with the available theoretical and experimental data[11]. For gold, a similar assessment of the numerical conver-

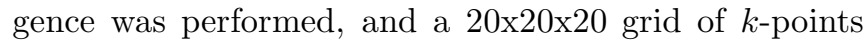
was found to show a good numerical convergence for the 32 atom cell.

We have calculated the $\varepsilon_{2}(\omega)$ at $T_{\text {ion }}=300 \mathrm{~K}$ for two different electron temperatures, $T_{\text {ele }}=300 \mathrm{~K}$ and $T_{\text {ele }}=27851 \mathrm{~K}(2.4 \mathrm{eV})$, corresponding to before and immediately after the incident pump laser with the absorbed energy density of $E_{D}=2.9 \mathrm{MJ} / \mathrm{kg}$ (see Fig. 11). As discussed in Sec. III, the measured $\varepsilon_{2}(\omega)$ shows an enhancement and red-shift of the interband transition peak, which keeps its shape for up to $t=4$ ps (steady state) at this $E_{D}$; so-called quasi steady state (QSS) behavior. As seen in Fig. 11, the theoretical $\varepsilon_{2}(\omega)$ at $T_{e l e}=2.4 \mathrm{eV}$ does not exhibit a discernible interband transition peak at all, resembling instead a Drude-like monotonic decrease. From a theoretical viewpoint, considering the assumed thermal equilibrium of the electrons (i.e. the Fermi distribution function with the high electron temperature, $T_{\text {ele }}=2.4 \mathrm{eV}$ ), it is naturally expected that the $\varepsilon_{2}(\omega)$ behaves similarly to the Drude term.

In general, the interband transition peak of gold, at $\omega=\omega_{0}$, in $\varepsilon_{2}(\omega)$, originates from pairs of bands satisfying the following conditions: they are nearly parallel

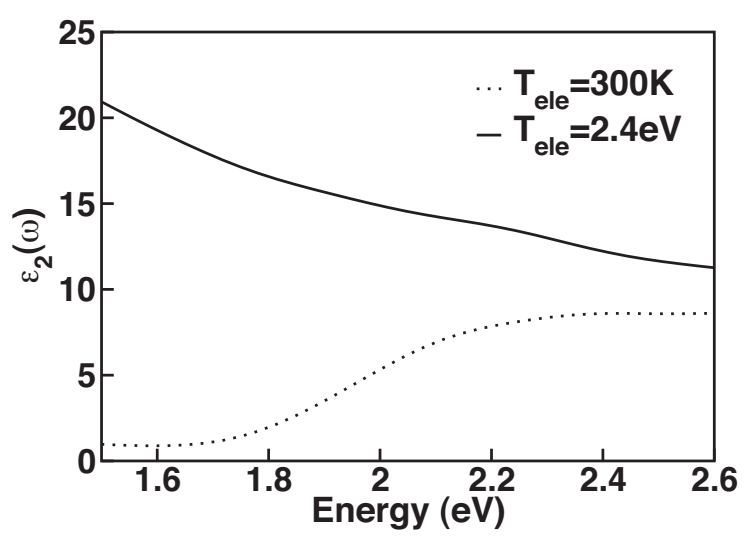

FIG. 11: Imaginary part of dielectric function of $f c c$ gold at $T_{\text {ele }}=300 \mathrm{~K}$ and at $T_{\text {ele }}=2.4 \mathrm{eV}$. The same atomic configuration generated by the $\mathrm{MD}$ at $T_{i o n}=300 \mathrm{~K}$ was used.

and separated by approximately $\omega=\omega_{0}$, they satisfy the dipole selection rule (the angular momentum character of the final state differs from that of the initial state by unity), and the band occupations have a non-zero difference (at low temperatures, they will lie on opposite sides of the Fermi energy). We note that in the case of gold, the band structure is complex (see Fig. 9) and parallel bands are realized only in small volumes of the FBZ (unlike aluminum). Instead, the onset of the interband transition peak at ambient temperatures is determined by the dipole selection rule, marking excitations from the $5 d$ occupied bands to unoccupied states with $6 p$ character, and so $\omega_{0}$ effectively marks the position of the uppermost d-bands below the Fermi level. Furthermore, as the electron temperature is elevated, and the Fermi distribution function deviates more from a step-function, smearing of the interband transition peak is observed. This is in contrast to the case of aluminum, since the parallel bands span a wide energy range and the interband transition peak persists even at high $T_{\text {ele }}[11]$. Therefore, raising $T_{\text {ele }}$ of gold would smear out the interband transition peak much sooner than it would for aluminum. In fact, this is what we obtained in calculations: the interband transition peak of gold at around $3 \mathrm{eV}$ is completely smeared out at $T_{\text {ele }}=2.4 \mathrm{eV}$, which is not consistent with the feature observed in QSS. This strongly suggests that some of the assumptions used to calculate the $\varepsilon_{2}(\omega)$ are not valid for analyzing the experimental results. Next, we will explore a possible cause of this discrepancy.

One might speculate that for high laser intensity, the excited electrons might never reach an equilibrated Fermi distribution, and this might be responsible for the QSS behavior. Although we do not completely exclude this possibility, the non-Fermi occupation alone seems unlikely to provide an explanation for the QSS behavior based on the following observations. Generally speaking, the electron-electron scattering time scale is inversely 
proportional to the excitation energy. The thermalization time scale of electrons in gold foil has been measured by time-resolved photo-emission measurements, and it was reported that the electron occupation equilibrates to a Fermi distribution within several hundred femtosecond [33]. In that study, relatively low pump laser intensities were used in the experiments (roughly 1/20-th of the damage threshold [27] for a gold foil), while our study focuses on higher pump laser intensity. Therefore, the above arguments rather suggest that the thermalization time scale of electrons will be significantly shorter than the QSS duration, $\tau_{Q S S}=4 \mathrm{ps}$ for $E_{D}=2.9 \mathrm{MJ} / \mathrm{kg}$. Recent time resolved UED measurements showed monotonic increase of thermal disorder in the lattice [15], where a range of pump laser fluences comparable to our experiments were explored $\left(E_{D}=1.1 \sim 2.85 \mathrm{MJ} / \mathrm{kg}\right)$. In their experiments, the estimated melting time scale behavior qualitatively agreed with the duration of QSS behavior (see Fig.5), suggesting that the states of gold foils in those experiments are essentially equivalent, however, these experiments measure the dynamics of the lattice[15] while ours measures the dynamics of an optical property[8]. If non-Fermi distributions of electron populations are responsible for the QSS behavior, the electrons would have to keep relaxing in such a way that it does not change the profile of $\varepsilon_{2}(\omega)$, while the lattice disorder is monotonically increasing, which does not seem to be plausible.

While we re-examined the details of our calculation procedure, we found a potentially problematic assumption that has been used. At $T_{\text {ele }}=2.4 \mathrm{eV}$ (corresponding to $E_{D}=2.9 \mathrm{MJ} / \mathrm{kg}$ ), the tail of the Fermi distribution function far exceeds the workfunction of gold $(\sim 5 \mathrm{eV})$ [34-36] (See Fig. 10). Therefore, electrons having higher energy than workfunction should be ejected from the gold foil. The ejection of electrons due to a pulse laser is very well known[37]. It is also known that the ejection of electrons will charge up the gold foil, and consequently, the workfunction will increase. It is generally considered that the positive charge of the sample will pull back the ejected electrons, and the electrons will stay near the gold foil as a layer of electron gas, which is refered to as the electronic sheath. However, we were not able to find any information in the literatures that would allow us to determine the amount of ejected electrons for a given $E_{D}$ and for a given geometry (thickness) of gold foil, nor the relaxation time scale for the ejected electrons to return to the gold foil. Here we will tentatively make the following hypothesis: The ejected electrons do not return to the gold foil within the QSS duration, therefore the gold foil can be temporarily positively charged, and the $T_{\text {ele }}$ within the gold foil can be significantly lower than the estimate without considering the electron ejection. Next, we examine whether this charged gold exhibits the characteristic feature of $\varepsilon_{2}(\omega)$ of QSS, namely, the red-shift and enhancement of the interband transition peak.

Using exactly the same atomic configuration used to calculate the $\varepsilon_{2}(\omega)$ in the Fig. $11, \varepsilon_{2}(\omega)$ of positively charged gold at two electron temperatures, $T_{\text {ele }}=300 \mathrm{~K}$

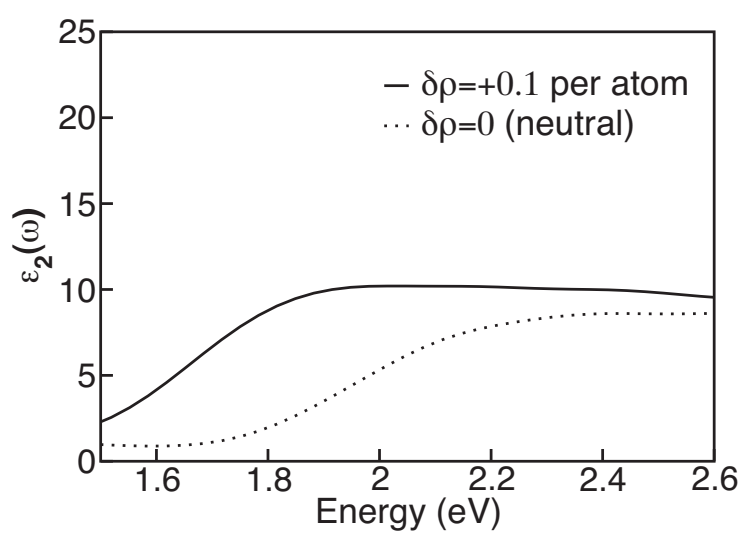

FIG. 12: The imaginary part of dielectric functions of $f c c$ gold at $T_{i o n}=T_{\text {ele }}=300 \mathrm{~K}$ : Positively charged $(\delta \rho=+0.1$ per atom) and neutral.

and $T_{\text {ele }}=2.4 \mathrm{eV}$, was calculated (see Fig. 12). It can be seen that, at low electron temperature, the positively charged gold exhibits the enhancement and red-shift in the interband transition peak, the characteristic feature of $\varepsilon_{2}(\omega)$ during the QSS. The mechanisms that yields this red-shift and enhancement can be easily understood in the following way. First, the positive charge is realized by removing conduction electrons, which corresponds to lowering the Fermi level below that of the neutral case. Since the interband transition peak originates from excitations of the occupied $d$-band to the unoccupied $p$-band, and the $d$-band has a relatively flat dispersion compared to the unoccupied $p$-band (see Fig. 9), lowering the Fermi level will lower the transition onset (see Fig. 9, and eq. $1)$. Since there is a prefactor, $1 / \omega^{2}$, to the transition matrix elements in the Kubo-Greenwood formula (see eq. 1), decreasing the transition onset (red shift of the interband transition peak) will enhance the peak height. At high electron temperature, the interband transition peak of the positively charged gold can be smeared out for the same reason as with the neutral case, which is seen in the calculated $\varepsilon_{2}(\omega)$ for high $T_{\text {ele }}$ (see Fig. 13). Therefore, we reach to the following conclusion: If the $T_{\text {ele }}$ is, in fact, much lower than the expected value, $T_{\text {ele }}=2.4 \mathrm{eV}$, the positive charge state of gold foil could account for the enhanced and red-shift in the peak of $\varepsilon_{2}(\omega)$ associated with the QSS. Therefore, as one possible explanation for the QSS behavior, we tentatively propose the following scenario. A femtosecond pulse laser with sufficiently high $E_{D}$ (on the order of one $\mathrm{MJ} / \mathrm{kg}$ ) ejects a significant amount of conduction electrons (e.g. on the order of 0.1 electron per one gold atom) from the gold foil $(\mathrm{d}=30 \mathrm{~nm}$ or less), and a significant portion of deposited energy might be carried away by the ejected electrons, which might lead to an unexpectedly low electron temperature within the foil. Since the ejected electrons are physically separated from the gold foil, they would not scatter the 


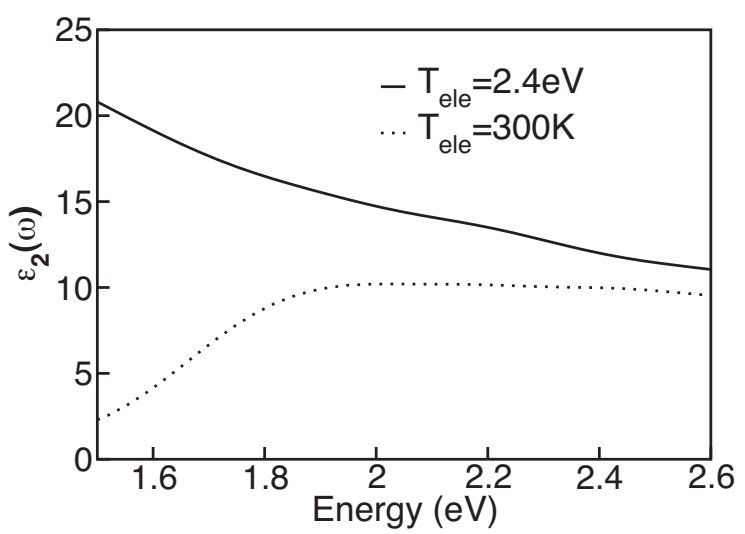

FIG. 13: The imaginary part of dielectric functions of ionized $(\delta \rho=+0.1$ per atom $) f c c$ gold at $T_{i o n}=300 \mathrm{~K}$ with different electron temperatures: $T_{\text {ele }}=300 \mathrm{~K}$ and $T_{\text {ele }}=2.4 \mathrm{eV}$.

electrons and the phonons in the gold foil as quickly as the electrons within the foil would. This would effectively slow down the heating of the lattice.

In the aforementioned UED measurements on the pumped gold foil, the monotonic change of Debye-Waller factor was reported, which corresponds to a monotonic increase of thermal disorder[15]. The development of thermal disorder continues until the liquid signal dominates its diffraction pattern, from which they concluded that a gold foil pumped with a femtosecond laser pulse exhibits thermal melt,[15] as opposed to semiinstantaneous non-thermal melt observed with pumped semiconductors[38]. They found that the rate of change in DWF is significantly slower than the estimate by the two-temperature model with temperature dependent electron-phonon coupling[39]. They attributed the slow rate of change in DWF to the lattice phonon hardening due to the high electron temperature[40], which leads to the slower rate of increase in the amplitude of lattice vibration[15]. This interpretation, consistent within their measurements, conflicts with the broadband $\varepsilon_{2}(\omega)$ measurements since such a high electron temperature leads to a Drude like $\varepsilon_{2}(\omega)$ (see Fig. 11), while enhanced and red-shifted of the interband transition peak in $\varepsilon_{2}(\omega)$ was observed.

Our interpretation discussed before turned out to be consistent with both of those experiments[8, 15]. If large amount of electrons are ejected with high kinetic energy, it is perhaps possible that the majority of ejected electron might not be able to return to the gold foil during such a short period of time, which will slow down the lattice heating and the rate of change in the DWF.

At last, to examine the validity of our hypothetical interpretation, a more quantitative characterization on the dynamics of ejected electrons is absolutely needed, and we call for further theoretical/ experimental investigations on this issue. Nevertheless, the missing inter- band transition peak at $T_{\text {ele }}=2.4 \mathrm{eV}$ presented in Fig 11 indicates that, at a high $E_{D}$, significantly higher than the damage threshold, an extra caution in determining the initial electronic state (or $T_{\text {ele }}$ ) is absolutely needed, which ultimately dictates the following dynamics of the system.

\section{MODEL OF ELECTRON SHEATH, A FINITE TEMPERATURE THOMAS-FERMI APPROACH}

As it was discussed in the previous section, the presence of surface (vacuum next to the gold foil) was omitted in the $a b$ initio calculations of $\varepsilon_{2}(\omega)$. Unfortunately, an ab initio simulation of the entire system (30nm gold foil with vacuum at both sides) is not possible at this moment due to unrealistic computational cost. In this section, we introduce a model that assumes electronic thermal equilibrium in a background of fixed ions after a prompt laser excitation to investigate the surface effect on the $\varepsilon_{2}(\omega)$ of gold foil with a comparable geometry to the real sample. This model provides a detailed description of the electronic structure near the interface of the metal (Gold) and vacuum (electron sheath) at high temperatures.

The model presented is justified if the time scales involved are short enough so that the atoms do not have time to move from their lattice positions, but large enough for electrons (which have a much faster dynamics) to reach a state of thermal and electrostatic equilibrium. It also includes other specific approximations: the jellium background model, rigid (temperature independent) band structures and the Thomas-Fermi self consistent field approximation. Once these approximations are established we solve for a one dimensional model of the metal-vacuum interface. Asymptotic behavior of electron density is obtained numerically and analytically. A complete model for the electron sheath is therefore obtained.

The use of this particular self-consistent model is inspired in the observation that for bulk density of states at high temperature, an appreciable fraction of electrons have energies above the work function (see Fig. 10). In principle, this gives the impression that such fraction of electrons will escape the material throughout the metal surface to a far away region in the vacuum. This is a possible picture, however, it is not an equilibrium state, since the sample will have a net positive charge that will tend to bring those electrons back. The Coulomb force has a very long range of action, therefore it is unlikely that a macroscopic number of electrons can leave the material without compensation. In its turn, the electrons that remain close to the surface will screen the electric field. Eventually there will exist a stationary charge distribution that will allow the electrons to have a thermal (Fermi) distribution and, at the same time, reach electrostatic equilibrium.

This conceptual picture calls for the need of solving this problem self-consistently with a coupled electrostatic potential. Thomas-Fermi (TF) model is one of the sim- 
Gold

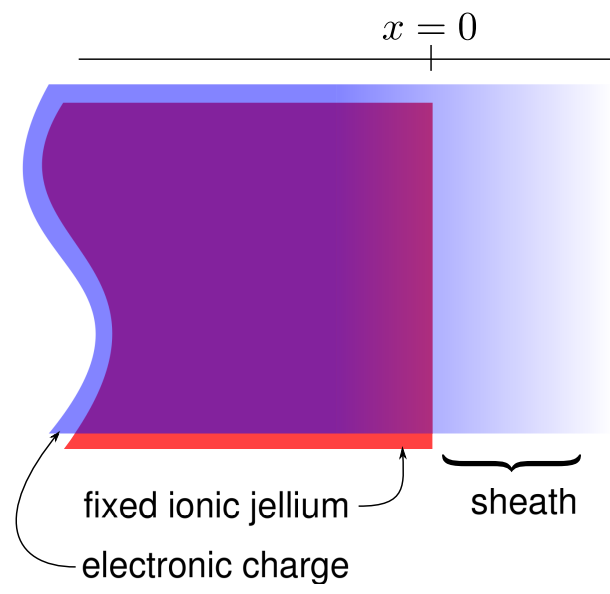

FIG. 14: (color online) Planar model of metal/vacuum interface. Ions are fixed $\left(T_{\text {ion }}=0\right)$ and approximated as jellium (uniform background of positive charge in the Gold region). Electronic density is dynamic, its stable distribution controlled by thermal electronic equilibrium and electrostatic self consistency. The electronic charge in the vacuum region is called electron sheath. In the Gold and vacuum region, electrons have different equilibrium densities of state.

plest descriptions that takes into account the described self-consistency. The TF model is a quantum mechanical description of the electronic structure based on the approximation that at each point in space the electronic state is determined by the density [41-43]. TF can describe electrostatically self-consistent electronic densities, without invoking fitting parameters and with a minimal computational cost it can give a good idea of the electronic density features at many different scales.

The ionic background is regarded as a jellium of positive charge (Fig. 14). This allows us to assert that a macroscopic electrostatic potential is a function of the unbalanced charge $\delta n$, with respect to the zero temperature charge distribution $\left(\delta n(\mathbf{r})=n(\mathbf{r})-n_{0}\right)$ via a Poisson equation

$$
\nabla^{2} V(\mathbf{r})=-4 \pi e^{2} \delta n(\mathbf{r}) .
$$

Within the TF approximation the net electrostatic potential itself enters in combination with the chemical potential producing a modulation of the density in space:

$$
n(\mathbf{r})=\int D_{\mathbf{r}}(E) \frac{1}{1+e^{\frac{E+V(\mathbf{r})-\mu}{k_{\mathrm{B}} T_{\text {ele }}}}} \mathrm{d} E
$$

This is based on the assumption that a meaningful density of states can be defined in regions where $V$ varies slowly. If this is the case, we can define an electronic density as a function of the local density of states (LDOS) $D$. The hypothesis of thermal equilibrium (well defined electronic temperature) enters through the Fermi function.
Finally, the chemical potential is obtained by constraining the conservation of charge

$$
\int \delta n(\mathbf{r}) \mathrm{d} \mathbf{r}=0
$$

The above three equations (2), (3) and (4) constitute the Thomas-Fermi equations at finite temperature.

Note that in the present variation of the TF equations we allow the density of states to be a function of the position. The local density of states $D$ is an external parameter to the model. To represent the metal/vaccum interface, we choose a discontinuous form of $D$ as a function of coordinates. Two different LDOS are used depending on the region of space

$$
D_{\mathbf{r}}(E)= \begin{cases}D^{\text {Gold }}(E) & \text { if } \mathbf{r} \in \text { Gold region } \\ D^{3 \mathrm{DEG}}(E) & \text { if } \mathbf{r} \in \text { vacuum region }\end{cases}
$$

In vacuum, a natural choice for the LDOS is the three dimensional free electron gas (3DEG) density of states[44]

$$
D^{3 \mathrm{DEG}}(E)=\frac{m}{\hbar^{3}} \frac{1}{\pi^{2}} \sqrt{2 m(E-W)}
$$

where $m$ is the electron mass.

In the metal region, $D^{\text {Gold }}$ is taken from a separate calculation of valence and conduction band structure calculations performed within the $a b$ initio DFT-LDA-PW framework (Section IV).

These densities of states are shown in Fig. 10. They have to be properly aligned in the energy scale. The experimental work function of Gold $W=4.8 \mathrm{eV}[35]$ is the parameter used to align the vacuum level (bottom of the free particle LDOS) with the Fermi energy $\left(E_{\mathrm{F}}\right)$ in the metal. We set the bottom of the 3DEG LDOS to $W\left(E_{\mathrm{F}}=0\right)$. Implicit is the assumption that the LDOS does not depend on the electronic temperature.

Details of derivation and solution of the model equations can be found in Appendix. The asymptotic solution of the density is found to decay polynomially at large distances in vacuum

$$
n(x)=\frac{k_{\mathrm{B}} T_{\text {ele }}}{2 \pi e^{2}\left(x-x_{0}\right)^{2}} .
$$

The physical interpretation that we envision is the following: At high temperatures, a fraction of electrons have energies above the work function, they are allowed to escape the material surface and leave it positively charged. These electrons do not travel far away due to the positively charged state of the metal, and stay close forming an electron sheath. The negatively charged electron sheath screens the positively charged material but only partially. As we go to distances farther away from the material there are less electrons available to compensate the remaining part of the charge and the screening becomes less effective. This allows electrons to be present in regions far from the surface, but always bound to the 

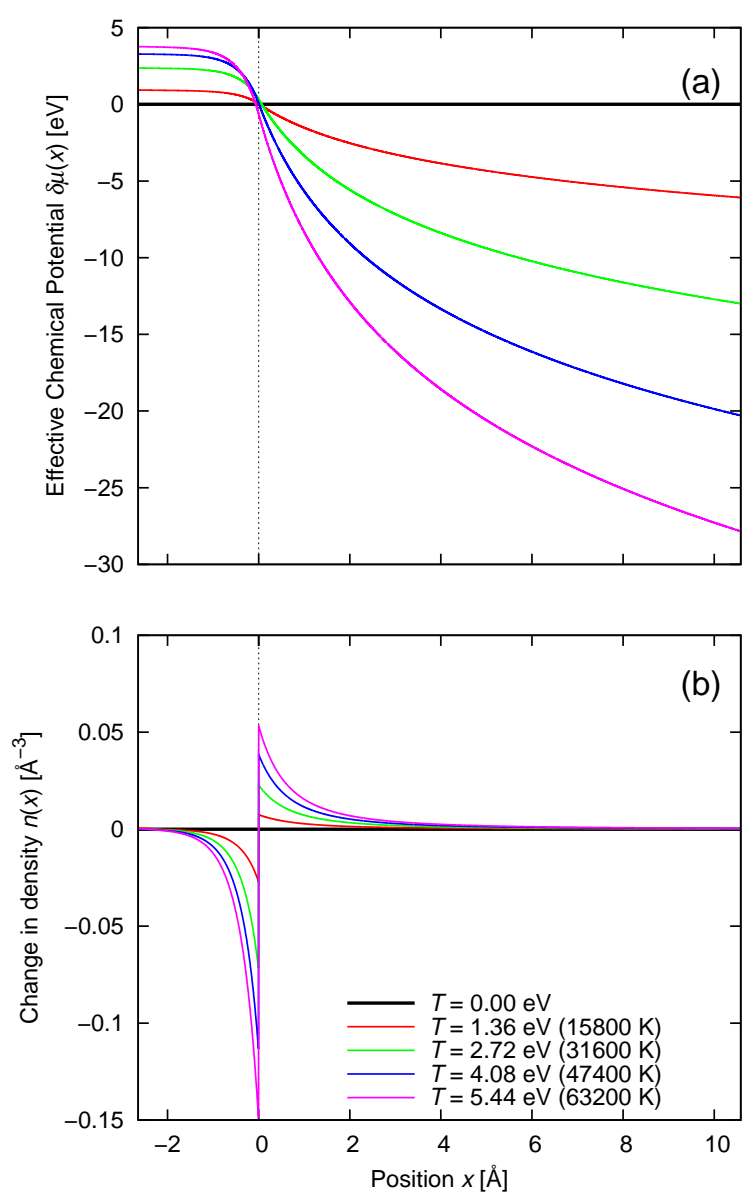

FIG. 15: Solution of the one dimensional Thomas-Fermi model resulting from the integration of the one dimensional equation at different temperatures. The vertical line $(x=0)$ represents the Gold/vacuum interface. (a) Self-consistent effective chemical potential $\delta \mu(x)$. (b) Net density inside the Gold region and in the vacuum (sheath). For comparison, in bulk Gold the equibrium density is $n_{0}=0.6485 \AA^{-3}$. At each temperature, the area below the curves at a given temperature add up to zero (charge conservation), on the left the decay is exponential on the right the decays is quadratic.

surface although with a very weak logarithmic potential. We expect the asymptotic solution to be valid at most up to distances where the planar approximation breaks down. In the experiment the heating laser hits a spot of finite size, this size also gives an idea of the distance at which the planar hypothesis stops being valid.

The one dimensional differential equation to solve is

$$
\delta \mu^{\prime \prime}(x)=4 \pi e^{2} I_{T}^{(x)}(\delta \mu(x))
$$

where

$$
I_{T}^{(x)}(\delta \mu)= \begin{cases}I_{T}^{\mathrm{Gold}}(\delta \mu) & x<0 \\ I_{T}^{3 \mathrm{DEG}}(\delta \mu) & x>0\end{cases}
$$

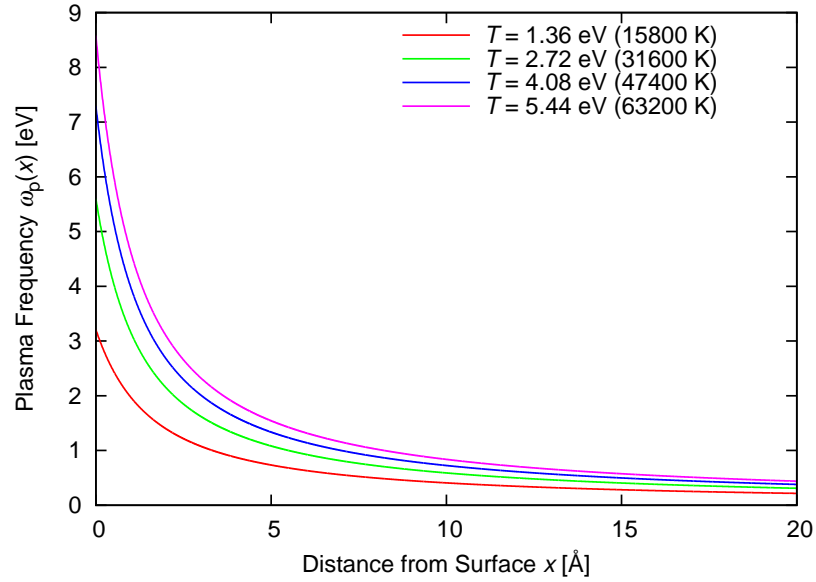

FIG. 16: Plasma frequency of the electron sheath as a function of distance $\omega_{\mathrm{p}}(x)$ at different temperatures.

are the thermal averaged integrated EDOS (see definition in Appendix), and charge conservation appears as boundary conditions

$$
\begin{aligned}
\delta \mu(-\infty) & =\delta \mu^{*}(T) \\
\delta \mu^{\prime}(+\infty) & =0
\end{aligned}
$$

The numerical method used to solve for $\mu(x)$ is a 4 th order Runge- Kutta. We perform the numerical integration in the range from -2.7 to $150 \AA$. The boundary conditions at the extremes are handled by the shooting method [45]. Solutions are shown in Fig. 15.

The model also predicts that, for this range of electronic temperatures, the charged state (electron depletion) in the metal decays exponentially fast, and that it is only confined to lengths smaller than the lattice parameter. This is the case as long as we assume thermal electronic equilibrium.

It is interesting to analyze the electron sheath formed near the surface in the vacuum region, for example, if we calculate the plasma frequency of the free electron gas based on the density $\omega_{\mathrm{p}}=\sqrt{4 \pi n e^{2} / m}$. The resulting plasma frequency as a function of distance $\omega_{\mathrm{p}}(x)$ in the vacuum region is shown in Fig. 16. The asymptotic solution in vacuum is

$$
\omega_{\mathrm{p}}(x) \sim \sqrt{\frac{2 k_{\mathrm{B}} T_{\text {ele }}}{m}} \frac{1}{x-x_{0}}
$$

This is a function that decays very slowly with distance. Using the Drude formula for the dielectric function of the electron sheath,

$$
\varepsilon_{e}(\omega)=1-\frac{\omega_{\mathrm{p}}^{2}}{\omega(\omega+i / \tau)}
$$

where $\tau$ is the electron collision time, and a matrix algorithm for dielectric function of multilayer films [46], we calculated the effect of the electron sheath on the reflec- 
tion and transmission of the probe in the experimental conditions. With a spatial cutoff of the sheath at $21 \AA$, and a large range of $\tau$ (from $1 \times 10^{-17} s$ to infinity), it turns out that the electron sheath based on this model has negligible effect on the optical properties.

This model is thermodynamic in essence. The model does not rule out that a (thermodynamically) microscopic but measurable number of electrons still can escape from the material. A different kind of limitation is imposed by the model itself. As density decays at large distances there is no mechanism for thermal equilibration and the TF no longer holds. The results presented in this section suggest that an assumption such as thermal electron used in the two temperature model needs to be carefully re-examined its validity for the range of $E_{D}$ explored in those experiments.

\section{SUMMARY}

To summarize, our FDI and broadband measurements have given us some interesting insights to the behavior of ultrafast laser excited gold. The quasi-steady state behavior, as observed in single-wavelength measurements, is confirmed for the whole visible region. It is found that the interband transition peak, an indirect evidence of the presence of $f c c$ lattice ordering, in the dielectric function is persistent throughout the quasi-steady state, which is consistent with the recent UED measurement on the pumped gold foil[15]. Our broadband $\varepsilon_{2}(\omega)$ measurements have shown the enhancement and red shift of the interband transition peak upon ultrafast laser heating.

The $\varepsilon_{2}(\omega)$ of a pumped gold calculated by DFT with thermal electrons assumption does not reproduce the enhancement and red-shift due to the smearing effect caused by the high electronic temperature $\left(T_{\text {ele }}=2.4 \mathrm{eV}\right.$ for $E_{D}=2.9 \mathrm{MJ} / \mathrm{Kg}$ ). On the other hand, a positively charged gold with colder electrons does exhibit the enhanced red-shift. Therefore, we propose a novel charge neutrality breakdown scenario to explain the observations. With such a high intensity of pump laser, a substantial amount of electrons might escape from the target with large kinetic energy and do not come back before the target melts and disassembles; the target might be kept positively charged significantly.

A simple model assuming thermalized electrons is shown to be able to describe the formation of a negatively charged electron sheath on the surface. However, the non-neutrality is confined to lengths smaller than the lattice parameter due to strong screening, and the sheath effect on the optical properties is negligible, which is not consistent with the experimental observation.

Above disagreement between theory and experiments suggests that the thermal electron assumption, used in the two-temperature model, needs to be carefully reexamined when the pump laser intensity far exceed the damage threshold, on the order of one $\mathrm{MJ} / \mathrm{kg}$ or higher for gold. We call for further theoretical/experimental investigations to study the mechanisms of the QSS behavior in the optical property of pumped gold foil.

\section{Acknowledgments}

We wish to thank L. X. Bendict, J. DuBois, P. Heimann and B. Cho for valuable discussions, and JLF staff for laser operation. This work was performed under the auspices of the U.S. Department of Energy by Lawrence Livermore National Laboratory under Contract DE- AC52-07NA27344, and was also supported by the Natural Sciences \& Engineering Research Council of Canada.

\section{Appendix A}

In Eqs. (1)-(5) the potential $V(x)$ and the chemical potential $\mu$ can be absorbed in an effective positiondependent chemical potential $\mu(x)=\mu-V(x)$. Since we are interested in changes with respect to the uniformly neutral bulk, we want to concentrate in changes of the chemical potential with respect to the Fermi energy $\delta \mu(x)=\mu(x)-E_{\mathrm{F}}$. (We know a priori that at $T=0$ there is no macroscopic potential $V(x)=0$ and that $\mu(x)=E_{\mathrm{F}}$.)

This change of variables allows us to simplify the set of equations into

$$
\nabla^{2} \delta \mu(\mathbf{r})=4 \pi e^{2} I_{T}(\delta \mu(\mathbf{r}))
$$

plus boundary conditions resulting from Eq. 4. Where we define

$$
I_{T}(\delta \mu)=\int D(E) \frac{1}{1+e^{\frac{E-E_{\mathrm{F}}-\delta \mu}{k_{B} T}}} d E-n^{0} .
$$

This function can be tabulated for each given density of states (3DEG or Gold), and at a given temperature.

In the case of $3 \mathrm{DEG}$ (Eq. 6 and $n_{0}=0$ ) the function can be calculated analytically:

$$
I_{T}^{3 \mathrm{DEG}}(\delta \mu)=\frac{m}{\hbar^{3}} \frac{1}{\pi^{2}} \sqrt{\frac{m \pi}{2}}\left(k_{\mathrm{B}} T\right)^{3 / 2} \mathrm{~F}_{1 / 2}\left(-\frac{W-\delta \mu}{k_{\mathrm{B}} T}\right)
$$

where $\mathrm{F}_{n}$ is the Fermi-Dirac function [47-49]. In the case of Gold $\left(n_{0}=0.6485 \AA^{-3}\right), I_{T}^{\text {Gold }}(\delta \mu)$ must be computed numerically from the ab initio $D(E)$. Fig. 17 shows these functions at different temperatures.

Note that in (A1) the resulting partial differential equation is non-linear and that we choose the potential $\delta \mu(\mathbf{r})$ as the main variable, the reason is that the potential must be a continuous variable. The density is not necessarily a continuous function at points where $D_{\mathbf{r}}$ is taken to be discontinuous.

For the problem of a planar metal/vacuum interface we can simplify the equations by assuming that the system 

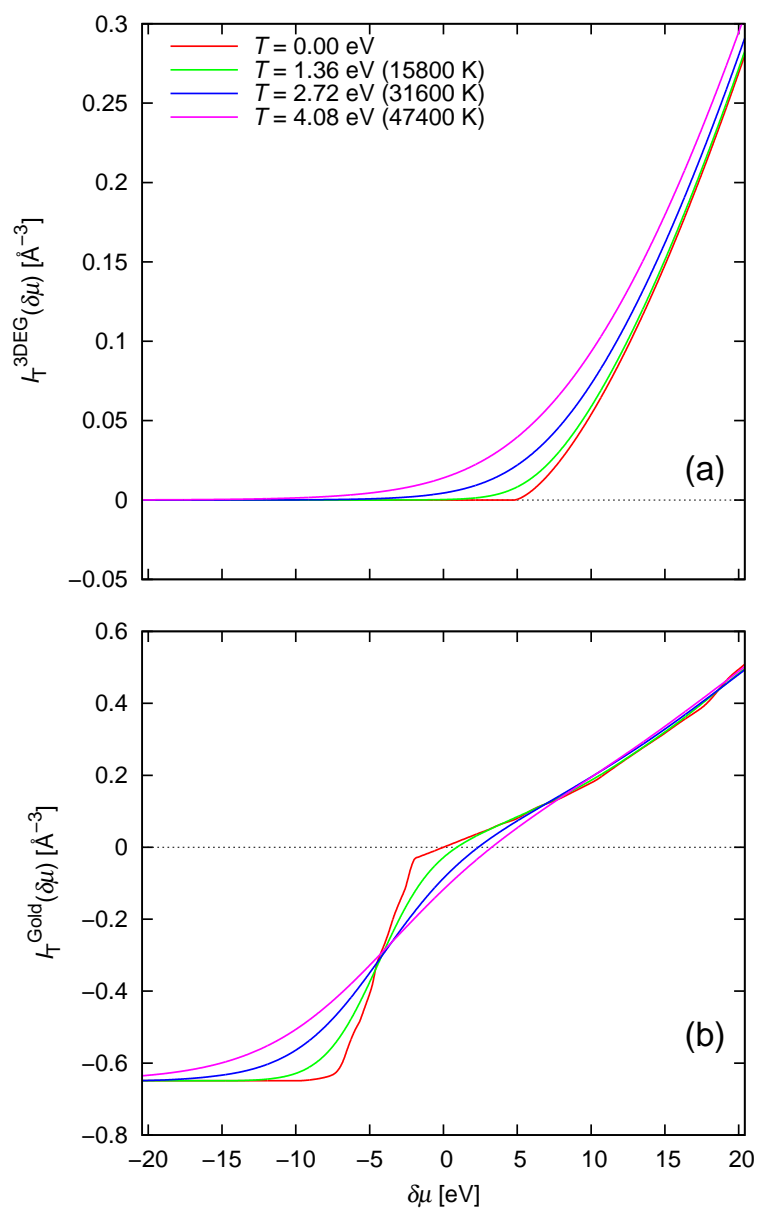

FIG. 17: Integral functions (a) for vacuum $I_{T}^{3 \mathrm{DEG}}$ and (b) for Gold $I_{T}^{\text {Gold }}$.

is one dimensional, i.e. the potential and the density only depend on the linear variable $x$. For $x<0$ we have the Gold region and for $x>0$ the vacuum (Fig. 14).

Before turning into the numerical solutions of the TF equations, it is convenient to analyze the asymptotic limits of the solution. Uniform solutions $\left(\delta \mu^{*}, \delta n^{*}\right)$ in large regions where the density of states is spatially uniform, have the condition

$$
I_{T}\left(\delta \mu^{*}\right)=0
$$

which naturally implies $\delta n^{*}=0$.

For Gold, $\delta \mu^{*}$ is well defined at each temperature by finding the roots of $I_{T}^{\text {Gold }}$ (see Fig. 17(b)). Incidentally, $\mu^{\text {Gold }}(T)=E_{\mathrm{F}}+\delta \mu^{*}(T)$ is the temperature-dependent chemical potential of bulk Gold.

By linearizing the differential equation (A1) around $\delta \mu^{*}$ we can show that the uniform limit is reached expo- nentially as a function of the position $\delta n(x) \propto e^{-\kappa|x|}$ with $\kappa=\sqrt{4 \pi e^{2} I_{T}^{\prime}\left(\delta \mu^{*}\right)}$. At zero temperature this coincides with the screening length of metal $\lambda=1 / \sqrt{4 \pi e^{2} D\left(E_{\mathrm{F}}\right)}$ [50]. Due to the high availability of electrons, the metal is very effective in screening the electric field. For Gold, $\lambda=0.58 \AA$.

In the vacuum region the situation is different, there is no uniform solution for finite values of $\delta \mu$ at $T>0$. That is, for $T>0, I_{T}^{3 \mathrm{DEG}}$ has no roots at finite values (see Fig. 17(a)). Therefore, in the vacuum region the effective chemical potential $\mu(\mathbf{r})$ decreases indefinitely.

This seems at first sight to be a contradiction, because if the system is neutral then the potential should tend to have zero gradient (no electric field at large distances). On the other hand, the potential diverges according to the previous argument. In one dimension, the only type of divergence that compatibilizes both expected behaviors, is the logarithmic divergence, which can be shown analytically as follows.

By taking the asymptotic limit of (A3) replaced in (A1), we obtain the non-linear differential equation

$$
\delta \mu^{\prime \prime}(x)=A e^{\delta \mu(x) / k_{\mathrm{B}} T}
$$

with $A=4 \pi e^{2} \frac{m}{\hbar^{3}} \sqrt{\frac{m \pi}{2}}\left(k_{\mathrm{B}} T\right)^{3 / 2} e^{-\frac{W}{k_{\mathrm{B}} T}}$. The solutions of this differential equation (parameterized by $c_{1}$ and $c_{2}$ ) are

$$
\delta \mu(x)=k_{\mathrm{B}} T \log \left(\frac{c_{1}}{A k_{\mathrm{B}} T\left(1-\cos \left(\frac{\sqrt{c_{1}} x+c_{2}}{k_{\mathrm{B}} T}\right)\right)}\right) .
$$

For $c_{1}<0$ the solutions are linearly divergent as a function of $x$, and for $c_{1}>0$ the solutions are periodic and divergent for finite values of $x$. None of these solutions correspond to a neutral system in a semi- infinite vacuum region.

There is only one class of solutions that does not diverge for finite values of $x$, and does not diverge linearly either, it is the one characterized by $c_{1} \rightarrow 0$ (and $\left.c_{2} / \sqrt{c_{1}} \rightarrow x_{0}\right)$. In this limit the solution is

$$
\delta \mu(x)=k_{\mathrm{B}} T \log \left(\frac{2 k_{\mathrm{B}} T}{A\left(x-x_{0}\right)^{2}}\right) .
$$

Replacing back $A$, we obtain the full asymptotic solution:

$$
\delta \mu(x) \sim W-k_{\mathrm{B}} T \log \left(\pi e^{2} \frac{m}{\hbar^{3}} \sqrt{m 2 \pi} \sqrt{k_{\mathrm{B}} T}\left(x-x_{0}\right)^{2}\right)
$$

for large $x$ in vacuum. Therefore the electric field decreases very slowly with distance. In contrast with the metal region, the vacuum region is very inefficient at screening the charged surface. 
Foord, Laser \& Particle Beams 23, 527 (2005).

[3] T. R. Dittrich et al., Phys. Plasmas 6, 2164 (1999).

[4] D. Saumon, G. Chabrier, D. J. Wagner and X. Xie, High Pressure Res. 16, 331 (2000).

[5] A. Forsman, A. Ng, G. Chiu and R. M. More, Phys. Rev. E 58, R1248 (1998).

[6] K. Widmann, T. Ao, M. E. Foord, D. F. Price, A. D. Ellis, P. T. Springer and A. Ng, Phys. Rev. Lett. 92, 125002 (2004).

[7] T. Ao, Y. Ping, K. Widmann, D. F. Price, E. Lee, H. Tam, P. T. Springer and A. Ng, Phys. Rev. Lett. 96, 055001 (2006).

[8] Y. Ping, D. Hanson, I. Koslow, T. Ogitsu, D. Pendergast, E. Schwegler, G. Collins and A. Ng, Phys. Rev Lett. 96, 255003 (2006).

[9] K. S. Trainor, J. Appl. Phys. 54, 2372 (1983).

[10] L. X. Benedict, J. E. Klepeis and F. H. Streitz, Phys. Rev. B 71, 064103 (2005).

[11] T. Ogitsu, L. X. Benedict, E. Schwegler, E. W. Draeger, and D. Prendergast, accepted in Phys. Rev. B.

[12] S. Mazevet, J. Clerouin, V. Recoules, P. M. Anglade and G. Zerah, Phys. Rev Lett. 95, 085002 (2005).

[13] J. Hohlfeld, S. S. Wellershoff, J. Gudde, U. Conrad, V. Jahnke and E. Matthias, Chem. Phys. 251, 237 (2000).

[14] P. Drude, Ann. Phys. (Leipzig) 1, 566 (1900).

[15] R. Ernstorfer, M. Harb, C. T. Hebeisen, G. Sciaini, T. Dartigalongue, and R. J. Dwayne Miller, Science 323, 1033 (2009).

[16] Y. Ping, D. Hanson, I. Koslow, T. Ogitsu, D. Pendergast, E. Schwegler, G. Collins and A. Ng, Phys. Plasmas 15, 056303 (2008).

[17] P. B. Johnson and R. W. Christy, Phys. Rev. B 6, 4370 (1972).

[18] A.G. Mathewson and H.P. Meyers, J. Phys. F: Met. Phys. 2, 403 (1972).

[19] P. S. Wehner, J. Stohr, G. Apai, F. R. McFeely, and D. A. Shirley, Phys. Rev. Lett. 38, 169 (1977).

[20] M. I. Kaganov, I. M. Lifshitz and L. V. Tanatorov, Soviet Physics JETP 4, 173 (1957).

[21] P. Hohenberg and W. Kohn, Phys. Rev. 136, B864 (1964); W. Kohn and Sham, Phys. Rev. 140, A1133 (1965).

[22] D. M. Ceperley and B. J. Alder, Phys. Rev. Lett. 45, 566 (1980); J.P. Perdew and A. Zunger, Phys. Rev. B 23, 5048 (1981).

[23] N. Troullier and J.L. Martins, Phys. Rev. B 43, 1993 (1991).

[24] L. Kleinman and D. M. Bylander, Phys. Rev. Lett. 48, 1425 (1982).

[25] The fhi98PP program package was used to generate all the pseudopotentials used in this work. See http://www.fhi-berlin.mpg.de/ th/fhi98md/fhi98PP/ for the detail of this program package.

[26] QUANTUM-ESPRESSO is a community project for high-quality quantum-simulation software based on density-functional theory and coordinated by Paolo Giannozi (see http://www.quantum-espresso.org and http://www.pwscf.org).

[27] J. Güdde, J. Hohlfeld, J. G. Müller, E. Matthias, Applied Surface Science 127-129, 40 (1998).

[28] F. Gygi, QBOX is a $\mathrm{C}++/ \mathrm{MPI}$ scalable parallel implementation of first-principles molecular dynamics (FPMD) based on the plane-wave pseudopotential formalism developed by (http://eslab.ucdavis.edu/).

[29] D.J. Chadi and M.L. Cohen, Phys. Rev. B 8, 5747 (1973).

[30] R. Kubo, J. Phys. Soc. Jpn. 12, 570-586 (1957); D. A. Greenwood, Proc. Phys. Soc. (London) 71, 585-596 (1958).

[31] D. Prendergast and S. G. Louie, arXive:0908.3876v2.

[32] E.L. Shirley, Phys. Rev. B 54, 16464 (1996).

[33] W.S. Fann, R. Storz, H. W. K. Tom, and J. Boker, Phys. Rev. B 46, 13592 (1992).

[34] W. M. H Sachtler, G. J. H. Dorgelo.

[35] P. A. Anderson, Phys. Rev. 115, 553 (1959).

[36] N. D. Lang and W. Kohn, Phys. Rev. B 3, 1215 (1971).

[37] S. I. Anisimov, B. L. Kapeliovich, and T. L. Perel'man, Sov. Phys. JETP 39, 375 (1974); F. Banfi et al., Phys. Rev. Lett. 94, 037601 (2005); G. Ferrini et al., Phys. Rev. Lett. 92, 256802 (2004); A. Apolonski et al., Phys. Rev. Lett. 92, 073902 (2004); G. Banfi, G. Ferrini, M. Peloi, and F. Parmigiani, Phys. Rev. B 67, 035428 (2003); N. Georgiev, D. Martinotti, and H.-J. Ernst, Phys. Rev. B 75, 085430 (2007); M. Aeschlimann et al., J. Chem. Phys. 102, 8606 (1995); K. L. Vodopyanov, L. A. Kulevskii, Cs. Tóth, Gy. Farkas, and Z. Gy. Horvàth, Appl. Phys. B 48, 485 (1989); C. Tóth, G. Farkas, and K. L. Vodopyanov, Appl. Phys. B 53, 221 (1991); C. Giannetti et al., Eur. Phys. J. B 53, 121 (2006); A. N. Belsky et al., Appl. Phys. B 78, 989 (2004).

[38] A. Rousse et al., NATURE 410, 65 (2001); A. M. Lindenberg et al., SCIENCE 308, 392 (2005); M. Herb et al., Phys. Rev. Lett. 100, 155504 (2008).

[39] Z. Lin and L. V. Zhigilei, V. Celli, Phys. Rev. B 77, 075133 (2008).

[40] V. Recoules, J. Clèrouin, G. Zèrah, P. M. Anglade, and S. Mazevet, Phys. Rev. Lett. 96, 055503 (2006); F. Botin and G. Zèrah, Phys. Rev. B 75, 174114 (2007).

[41] L. H. Thomas, in Mathematical Proceedings of the Cambridge Philosophical Society (1927), vol. 23.

[42] E. Fermi, Rend. Accad. Naz. Lincei 6, 32 (1927).

[43] Robert G. Parr and Yang Weitao, Density-Functional Theory of Atoms and Molecules (International Series of Monographs on Chemistry), Oxford University Press, USA, 1994.

[44] E. N. Economou, Green's functions in quantum physics, Springer Berlin, 1996.

[45] Press, W.H. and Teukolsky, S.A. and Vetterling, W.T. and Flannery, B.P., Numerical recipes: the art of scientific computing, Cambridge university press, 2007.

[46] M. Born and E. Wolf, Principles of Optics, 6th ed., Pergamon Press, Oxford, 1980.

[47] Galassi, M. and Davies, J. and Theiler, J. and Gough, B. and Jungman, G. and Booth, M. and Rossi, F., GNU Scientific Library Reference Manual-Revised Second Edition (v1. 8), Network Theory Ltd, (2006).

[48] E. W. Weisstein, Fermi-Dirac Distribution, From MathWorld-A Wolfram Web Resource (2009).

[49] E. W. Weisstein, Polylogarithm, From MathWorld-A Wolfram Web Resource (2009).

[50] J. M. Ziman, Principles of the Theory of Solids, Cambridge Univ Press, 1979. 